\title{
Besondere Abkürzungen
}

\author{
$A B C$ \\ abl. \\ afd \\ AfP \\ AM \\ Am. Jur. \\ Amtsbl. Saar \\ Ann. Rep. \\ Anw.-BI. \\ AöR \\ ArchPR \\ ARD
}

Ariz. L. Rev.

Ark. L. Lev.

BadenGVBI.

bad.-württ. PresseG

Bay-GVBI.

BayLT-Drucks.

BayObLG

BayObLGZ

BB

Berl. GVBI.

berl. PresseG

Beschl.

BGB

BGBI.

BGH

$B R$

BRD

Brem. GBI.

brem. PresseG

BT-Drucks.

BVerfG

BVerfGE

Cal. L. Rev.

CATV
American Broadcasting Corporation

ablehnend

Aktueller Fernsehdienst

Archiv für Presserecht (ab Januar 1971)

Amplitude Modulation (Mittelwelle)

American Jurisprudence (Cumulative Pocket Supplement 1970)

Amtsblatt des Saarlandes

Annual Report

Anwaltsblatt

Archiv des öffentlichen Rechts

Archiv für Presserecht, Beilage zu ZV + ZV

bis Dezember 1970

Arbeitsgemeinschaft der öffentlich-rechtlichen Rundfunkanstalten der Bundesrepublik Deutschland

Arizona Law Review

Arkansas Law Review

Badisches Gesetz- und Verordnungsblatt, Regierungsblatt der Landesregierung Baden

Gesetz über die Freiheit der Pesse (Baden-Württemberg)

Bayerisches Gesetz- und Verordnungsblatt

Drucksache des Bayerischen Landtags

Bayerisches Oberstes Landesgericht

Entscheidungen des Bayerischen Obersten Landesgerichts in Zivilsachen

Der Betriebsberater

Gesetz- und Verordnungsblatt für Berlin

Berliner Pressegesetz

Beschluß

Bürgerliches Gesetzbuch

Bundesgesetzblatt

Bundesgerichtshof

Bayerischer Rundfunk

Bundesrepublik Deutschland

Gesetzblatt der Freien Hansestadt Bremen

Gesetz über die Presse (Bremen)

Bundestagsdrucksache

Bundesverfassungsgericht

Entscheidungen des Bundesverfassungsgerichts

California Law Review

Community Antenna Television 
CBS

C. F. R.

3rd/7th Cir.

Col.

Col. L. Rev.

Cong.

Cong. Rec.

Conn.

Cornell L. Rev.

Cum. (Ann.) Pock. Suppl.
D. C.
D. C. Cir.
DDR
Diss.
DJZ
DLF
DOV
DRiz
DVBI.
DW

EGBGB
et al.
F. (2nd)
Fairness Primer)

FAZ

F. C. A.

F. C. B. J.

FCC

F. C. C. (2nd)

Fed. Reg.

F. H.

FM

FRC

F. R. C. Ann. Rep.

FuR

GA

GBI. Bad.-Württ.

Geo. L. J.

Geo. Wash. L. Rev.

GG

GKG
Columbia Broadcasting System

Code of Federal Regulations

U.S. Court of Appeal for the Third/Seventh Circuit

Colorado

Colorado Law Review

Congress

Congressional Records

Connecticut

Cornell Law Review

Cumulative (Annual) Pocket Supplement

Der Betrieb

District of Columbia

U. S. Court of Appeals, District of Columbia Circuit Deutsche Demokratische Republik

Dissertation

Deutsche Juristenzeitung

Deutschlandfunk

Die öffentliche Verwaltung

Deutsche Richterzeitung

Deutsches Verwaltungsblatt

Deutsche Welle

Einführungsgesetz zum Bürgerlichen Gesetzbuch und andere

Federal Reporter (Second series)

Applicability of the Fairness Doctrine in the Handling of Controversial Issues of Public Importance FCC-Public Notice vom 1. Juli 1964

Frankfurter Allgemeine Zeitung

Federal Code Annected (1969 Cumulative Pocket Supplement)

Federal Communications Bar Journal

Federal Communications Commission

Federal Communications Commission Reports

(Second series)

Federal Register, The National Archives of the United

States

Frankfurter Hefte

Frequency Modulation (Ultrakurzwelle)

Federal Radio Commission

Federal Radio Commission Annual Reports

Film und Recht

Goldtammers Archiv für Strafrecht

Gesetzblatt für Baden-Württemberg

Georgetown Law Journal

George Washington Law Review

Grundgesetz

Gerichtskostengesetz 
XXVIII

GMPI

GRUR

GS

GVBI. Schl.-H.

GVG

GV NW (bzW. GVBI, NW)

Hamb. GVBI.

namb. PresseG

Harv. J. Legis.

Harv. L. Rev.

Hess. GVBI.

hess. PresseG

House Hearings

House Staff Study

HR

H. R.

H. Rept.

III.

IVW

J. Law and Econ.

Journ.

Journ. Offic.

JR

JW

$\mathrm{JZ}$

KG

La. L. Rev.

LG

LPG

Md. L. Rov.

Mich. L. Rev.

Minn.

Minn. L. Rev.

Miss. Code Ann. 1942
Der zivilrechtliche Persönlichkeits- und Ehrenschutz in Frankreich, der Schweiz, England und den Vereinigten Staaten von Amerika; Gutachten des MaxPlanck-Institutes für ausländisches und Internationales Privatrecht (Dölle, Hrsg.)

Gewerblicher Rechtsschutz und Urheberrecht

Der Gerichtssaal

Gesetz- und Verordnungsblatt für Schleswig-Holstein Gerichtsverfassungsgesetz

Gesetz- und Verordnungsblatt für das Land Nordrhein-Westfalen

Hamburgisches Gesetz- und Verordnungsblatt

Hamburgisches Pressegesetz

Harvard Journal on Legislation

Harvard Law Review

Gesetz- und Verordnungsblatt für das Land Hessen

Hessisches Gesetz über Freiheit und Recht der Presse

Panel Discussion on the Fairness Doctrine and Related Subjects; Hearings before the Special Subcomittee on Investigations of the Committee on Interstate and Foreign Commerce U. S. House of Representatives

Legislative History of the Fairness Doctrine; Staff Study for the Committee on Interstate and Foreign Commerce U.S. House of Representatives

Hessischer Rundfunk

U.S. House of Representatives

U. S. House of Representatives Reports

Illinois

Informationsgemeinschaft zur Feststellung der Verbreitung von Werbeträgern

Journal of Law and Economics

Der Journalist

Journal Officie!

Juristische Rundschau

Juristische Wochenschrift

Juristenzeitung

Kammergericht

Louisiana Law Review

Landgericht

Landespressegesetz

Maryland Law Review

Michigan Law Review

Minnesota

Minnesota Law Review

Mississippi Code 1942 Annotated (1968 Cumulative Supplement) 


NAB
NBC
N. D. Lwyr.
NDR
Nev. Comp. Laws
Nds. GVBI.
nsd. PresseG
NJW
NRS
N. W.
nw. PresseG
N. Y.
N. Y. U. Intra. L. Rev.
OLG

$\mathrm{Pa}$.

PresseG

Pub. L.

RB

Report 1949

RGBI.

RGSt.

Rh.-Pf. GVBI.

rh.-pf. PresseG

RPG

R. R. (2nd)

RTNDA

S. Calendar schl.-h. PresseG

S. C. L. Rev.

S. Rept.

S. Ct. Miss.

SDR

Senate Staff Report

sess.

SFB

SGV NW

S. J. Res.

So. (2nd)

SR

Stat.

StGB

SWF
National Association of Broadcasters

National Broadcasting Corporation

Notre Dame Lawyer

Norddeutscher Rundfunk

Nevada Compiled Laws (1929)

Niedersächsisches Gesetz- und Verordnungsblatt

Niedersächsisches Pressegesetz

Neue Juristische Wochenschrift

Nevada Revised Statutes

North Western Reporter

Pressegesetz für das Land Nordrhein-Westfalen

New York

New York University Intramural Law Review

Oberlandesgericht

Pennsylvania

Pressegesetz

Public Law

\section{Radio Bremen}

FCC 'Editorializing by Broadcast Licensees', Report of Commission 1949; Appendix A zum Fairness Primer; zugleich 13 F. C. C. 1246 (1949)

Reichsgesetzblatt

Entscheidungen des Reichsgerichts in Strafsachen Gesetz- und Verordnungsblatt für das Land Rheinland-Pfalz

Landesgesetz über die Presse (Rheinland-Pfalz)

Reichsgesetz über die Presse

Pike and Fischer, Radio Regulations (Second Series)

Radio Television News Directors Association

U. S. Senate Calendar

Gesetz über die Presse (Schleswig-Holstein)

South Carolina Law Review

U. S. Senate Reports

Supreme Court of Mississippi

Süddeutscher Rundfunk

Fairness Doctrine; Staff Report prepared for the Subcommittee on Communications of the Committee on Commerce; U. S. Senate

session

Sender Freies Berlin

Sammlung des bereinigten Gesetz- und Verordnungsblattes für das Land Nordrhein-Westfalen

U. S. Senate Joint Resolution

Southern Reporter (Second Series)

Saarländischer Rundfunk

Statutes

Strafgesetzbuch

Südwestfunk 
Tenn. L. Rev.

Tex. L. Rev.

Trial

TV

U. Cinn. L. Rev.

U. Colo. L. Rev.

UFITA

UHF

U. Pitt. L. Rev.

U.S.

USA

U. S. C. A.

U. S. C. A. Const. Amend.

U. S. S. Ct.

$v$.

Va. L. Rev.

VHF

vol.

WDR

WRP

Yale L. J.

ZDF

$Z P O$

ZStW

$Z V+Z V$
Tennessee Law Review

Texas Law Review

Trial (American Trial Lawyers Association)

Television

University of Cincinnati Law Review

University of Colorado Law Review

Archiv für Urheber-, Film-, Funk- und Theaterrecht

Ultra High Frequency

University of Pittsburgh Law Review

United States; zugleich United States Supreme Court

Reports

United States of America

United States Code Annotated

. United States Code Annotated, Constitutional Amendments

United States Supreme Court

versus

Virginia Law Review

Very High Frequency

volume

Westdeutscher Rundfunk

Wettbewerb in Recht und Praxis

Yale Law Journal

Zweites Deutsches Fernsehen

Zivilprozeßordnung

Zeitschrift für die gesamte Strafrechtswissenschaft

Zeitungsverlag und Zeitschriftenverlag 